\title{
SASE-MAD protein structure determination and charge assignment to metal sites using XFEL crystallography
}

\author{
Asmit Bhowmick ${ }^{1}$, Aaron S. Brewster ${ }^{1}$, Nicholas K. Sauter ${ }^{1}$ \\ ${ }^{1}$ Molecular Biophysics and Integrated Bio-imaging Division, Lawrence Berkeley National Lab \\ Berkeley, California 94721
}

Email: abhowmick@lbl.gov

Advances in X-ray free-electron laser (XFEL) crystallography in recent years has enabled (a) protein structure determination by avoiding radiation damage and (b) time resolved studies of proteins ${ }^{1}$. Most XFEL protein structures currently are solved using Molecular Replacement (MR) that uses a reference protein structure, usually obtained at a synchrotron. There are however only a few instances of de novo structure determination at an XFEL facility, most of them using SAD and only one case using MAD ${ }^{2}$. Additionally, there is considerable interest in assigning charge states to metal sites in time resolved protein structures using XFEL that would help understand transition between different states. This is particularly important in studies of metalloenzymes and in principle can be done by analyzing the structure factor at the absorption edge of metal sites.

This work will present progress made in doing a simultaneous SASE-MAD structure determination and charge assignment by using the spread of the SASE spectra to get data at multiple wavelengths.

Specifically, I will elaborate on new strategies in the data reduction pipeline to index (i.e determine crystal orientations and miller indices) and integrate (i.e obtain intensities of Bragg spots) signal coming from different photon energies of the SASE spectra. Coupling these with new Bayesian estimation techniques for the structure factor of metal sites will allow us to assign a charge state to metal sites. I will also discuss software development in the context of CCTBX/DIALS packages ${ }^{3}$ for this project.

Abbreviations

XFEL: X-ray free-electron laser

SASE: Self-amplified spontaneous emission

SAD: Single wavelength anomalous dispersion

MAD: Multiple wavelength anomalous dispersion

LCLS: Linac Coherent Light Source

References

1. Sauter, N.K. and Adams, P.D., 2017. Overcoming Data Processing Challenges for Breakthrough Crystallography. In X-Ray Free Electron Lasers (pp. 70-87).

2. Gorel, A., Motomura, K., Fukuzawa, H., Doak, R.B., Grünbein, M.L., Hilpert, M., Inoue, I., Kloos, M., Kovácsová, G., Nango, E. and Nass, K., 2017. Multi-wavelength anomalous diffraction de novo phasing using a two-colour X-ray free-electron laser with wide tunability. Nature Communications, 8(1), p.1170.

3. Brewster, A.S., Waterman, D.G., Parkhurst, J.M., Gildea, R.J., Young, I.D., O'Riordan, L.J., Yano, J., Winter, G., Evans, G. and Sauter, N.K., 2018. Improving signal strength in serial crystallography with DIALS geometry refinement. Acta Crystallographica Section D: Structural Biology, 74(9), pp.877-894. 